Meta

Journal des traducteurs

Translators' Journal

\title{
Variation dénominative avec conséquences cognitives : quelques exemples détectés autour de " musée "
}

\section{Anna Joan Casademont}

Volume 63, numéro 2, août 2018

URI : https://id.erudit.org/iderudit/1055147ar

DOI : https://doi.org/10.7202/1055147ar

Aller au sommaire du numéro

\section{Éditeur(s)}

Les Presses de l’Université de Montréal

\section{ISSN}

0026-0452 (imprimé)

1492-1421 (numérique)

Découvrir la revue

\section{Citer cet article}

Joan Casademont, A. (2018). Variation dénominative avec conséquences cognitives : quelques exemples détectés autour de « musée ». Meta, 63(2), 444-466. https://doi.org/10.7202/1055147ar
Résumé de l'article

Cet article se veut une contribution à la description des phénomènes liés au caractère dynamique des discours spécialisés. Concrètement, nous y analysons la variation dénominative (avec conséquences cognitives) dans le domaine de la muséologie et de la muséographie dans un corpus en langue espagnole, et ce, pour les unités présentant le morphème muse(o)- extraites à l'aide de l'outil TermoStat (Drouin 2003). Pour ce faire, nous partons de la typologie de la variation dénominative proposée par Fernández-Silva (2013), ce qui nous permet de : (a) confirmer que la typologie est pertinente dans d'autres disciplines que celle pour laquelle elle a été créée, c'est-à-dire la pêche ; (b) relever et caractériser des tendances sur le plan de la variation, si elles existent, dans le domaine des musées. Cela permettra d'orienter postérieurement des choix lexicographiques vers l'information pertinente à expliciter. 


\title{
Variation dénominative avec conséquences cognitives: quelques exemples détectés autour de «musée»
}

\author{
ANNA JOAN CASADEMONT \\ Université TÉLUQ, Québec, Canada \\ anna.joan.casademont@teluq.ca
}

\section{RÉSUMÉ}

Cet article se veut une contribution à la description des phénomènes liés au caractère dynamique des discours spécialisés. Concrètement, nous y analysons la variation dénominative (avec conséquences cognitives) dans le domaine de la muséologie et de la muséographie dans un corpus en langue espagnole, et ce, pour les unités présentant le morphème muse(o)- extraites à l'aide de l'outil TermoStat (Drouin 2003). Pour ce faire, nous partons de la typologie de la variation dénominative proposée par Fernández-Silva (2013), ce qui nous permet de: (a) confirmer que la typologie est pertinente dans d'autres disciplines que celle pour laquelle elle a été créée, c'est-à-dire la pêche; (b) relever et caractériser des tendances sur le plan de la variation, si elles existent, dans le domaine des musées. Cela permettra d'orienter postérieurement des choix lexicographiques vers l'information pertinente à expliciter.

\begin{abstract}
This article aims at contributing to the description of phenomena related to dynamicity in specialised discourses. Specifically, we analyse denominative variation (with cognitive consequences) in museum studies and museography using Spanish language corpus. We consider units with the morpheme muse(o)- extracted using the tool TermoStat (Drouin 2003). For the analysis, we will use the denominative variation typology by Fernández-Silva (2013), which will allow us: (a) to confirm that the typology is adequate for disciplines other than fishing (for which it was created at the beginning); (b) to find and characterise possible tendencies of variation, in museum studies discourses, which will allow us to orientate subsequent lexicographic decisions related to the relevant information to be explicited.
\end{abstract}

\section{RESUMEN}

Este artículo quiere contribuir a la descripción de los fenómenos vinculados al carácter dinámico de los discursos especializados. Concretamente, analizamos la variación denominativa (con consecuencias cognitivas) en el ámbito de la museología y de la museografía en un corpus en lengua española; realizamos dicho análisis con las unidades que presentan el morfema muse(o)-, extraídas mediante la herramienta TermoStat (Drouin 2003). Para ello, partimos de la tipología de variación denominativa propuesta por Fernández-Silva (2013), que nos permite: (a) confirmar que la tipología es pertinente en disciplinas otras que la analizada inicialmente, es decir, la pesca; (b) detectar y caracterizar las tendencias a nivel de variación, si existen, en el ámbito de los museos. Ello permitirá orientar posteriormente las decisiones lexicográfias sobre la información pertinente que debe explicitarse.

\section{MOTS CLÉS/KEYWORDS/PALABRAS CLAVE}

terminologie, variation dénominative, muséologie, types de variations, facteurs de variation terminology, denominative variation, museum studies, types of variation, variation factors terminología, variación denominativa, museología, tipos de variación, factores de variación 


\section{1. État de l'art}

Devant les carences de la Théorie générale de la terminologie relativement au large éventail de nouvelles situations communicatives où la terminologie était utilisée, sont apparues au cours des vingt dernières années des voies alternatives dont l'objectif était de combler des vides descriptifs, explicatifs et appliqués: socioterminologie (Gaudin 2003, 2005), terminologie culturelle (Diki-Kidiri 2008), terminologie communicative (Cabré 1999, 2000), etc.

Un aspect clé caractérise toutes ces nouvelles approches: la reconnaissance de la variation (synonymie, polysémie, etc.) en tant que phénomène naturel du langage. Par le fait même, cette variation se manifeste donc dans les échanges spécialisés.

Le caractère dynamique de la langue mène ainsi au changement et permet d'expliquer l'existence de phénomènes comme la variation conceptuelle et la variation dénominative, que ce soit dans le discours spécialisé ou non. En terminologie, plusieurs approches visent à expliquer cet aspect dynamique et à le décrire: Cabré (1999, 2000, 2002, 2008), Temmerman (2000) et Faber et al. (2006, 2014), entre autres.

Ainsi, alors que la question de la variation en terminologie a été longtemps négligée, plusieurs des aspects de la variation sont aujourd'hui étudiés, et ce, à partir de différentes approches, dont: la variation des termes du point de vue textuel (Ciapuscio 1998), les traits caractérisant la variation dénominative (Freixa 2002, 2005, 2006), la variation conceptuelle à partir de la modulation sémantique du discours (Kostina 2011, 2014), la variation en tant que ressource de construction des connaissances dans les textes (Pecman 2012, 2014; Fernández-Silva 2016), la variation dénominative et le point de vue (Fernández-Silva 2013), la variation terminologique dans les discours oraux (Seghezzi 2013), la variation dénominative à partir de la motivation cognitive et communicative (Tercedor 2013) et la représentation de la variation contextuelle à partir de définitions (San Martín 2016).

Notre approche s'appuie notamment sur la Théorie communicative de la terminologie (TCT) proposée par Cabré (expliquée ci-dessus) qui considère que le caractère spécialisé des termes dépend de l'usage que l'on en fait dans le discours. Cette prémisse permet à Cabré de rendre compte des différents éléments de variation qui existent sur les plans cognitif et linguistique et d'étudier ainsi le caractère dynamique des productions spécialisées.

Afin d'améliorer notre compréhension du phénomène, il est nécessaire de décrire de façon systématique et détaillée la variation dénominative dans les productions communicatives des experts. Une telle description devrait nous permettre de tirer des constats généraux quant à l'apparition de certains phénomènes de variation dans les discours desdits experts. Nous pourrons ensuite nous pencher sur les facteurs relevant du dynamisme de la communication qui ont mené à cette variation. De cette façon, nous serons en mesure de comprendre, entre autres, comment les experts conceptualisent leur discipline.

Notre recherche a pour objectif de contribuer à la description des phénomènes liés au caractère dynamique dans les discours spécialisés à partir d'une analyse à l'appui de notre corpus.

Notre point d'amorce n'est donc pas nouveau dans le domaine: on peut constater dans le discours des experts une variation conceptuelle et dénominative, et cela est la conséquence du caractère dynamique des processus cognitifs de catégorisation et de transmission du savoir spécialisé. 
Fernández-Silva (2013: 12) définit la variation dénominative de la façon suivante: «fenómeno por el cual un concepto especializado es denominado a través de varias unidades terminológicas distintas, las cuales pueden diferir formalmente pero también semánticamente $»^{1}$. La définition de Fernández-Silva tient également compte du fait que la variation peut ne pas avoir de conséquences cognitives (les unités diffèrent formellement, mais pas sémantiquement), ou bien avoir de telles conséquences (quand le changement formel implique un changement sémantique). Dans ce dernier cas, les variantes reflètent différents aspects du contenu conceptuel sans que l'on considère qu'elles dénomment des concepts différents.

Dans le cadre de la TCT, nous partons de la classification de variation formelle de Freixa (2002) pour le domaine de l'environnement et de la typologie de la variation dénominative proposée par Fernández-Silva (2013) pour le domaine de la pêche, ce qui nous permettra de: (a) confirmer que la dernière typologie est pertinente dans d'autres disciplines; (b) relever et caractériser des tendances sur le plan de la variation, si elles existent, dans le domaine des musées.

Freixa (2002: 281) propose une classification formelle complète de la variation détectée dans le corpus d'environnement analysé dans sa recherche:

- Changements graphiques: terme et forme artificielle (symbole, formule chimique, etc.), terme et abréviation (sigle, etc.), changement orthographique.

- Changements morphosyntaxiques: avec maintien de la structure (changement de nombre, changement de genre, changement de préposition, etc.), avec changement de la structure ([Nom + Adjectif] et [Nom + Syntagme prépositionnel], monolexique et polylexique, etc.).

- Réductions: réductions de l'extension ([Nom + Adjectif/Syntagme prépositionnel/ Nom] et Nom, $[N+x+y]$ et $[N+x])$, réductions de la base ([Nom + Adjectif] et Adjectif nominalisé, etc.), autres réductions.

- Changements lexicaux: unités monolexicales, unités polylexicales (changement de base, changement d'extension, etc.).

- Différents changements complexes avec ou sans parenté formelle.

Fernández-Silva, à partir notamment des travaux de Freixa, propose une liste des types de variations avec conséquences cognitives. Sa typologie fondée sur un corpus s'appuie, comme nous l'avons mentionné ci-dessus, sur plusieurs exemples dans le domaine de la pêche:

- Changement de la configuration conceptuelle: quand la classe conceptuelle de la base, l'extension et la relation intraconceptuelle ne coïncident pas; par exemple, espèce capturée (faune, nature de l'objet) et produit de pêche (commercialisation, finalité pour l'homme).

- Variation dénominative dans la sélection de la catégorie: quand les bases des variantes ne coïncident pas; par exemple, territoire maritime et milieu marin; quand l'auteur parle de la mer conceptualisée comme un territoire appartenant à un État (territoire) ou comme un écosystème (milieu).

- Variation dénominative sur le plan de l'abstraction de la catégorie: quand les dénominations choisissent des concepts appartenant à la même classe conceptuelle, mais qui se trouvent dans des niveaux d'abstraction différents, c'est-à-dire hyperonymie et hyponymie; par exemple, professionnel, producteur et pêcheur, qui servent à refléter différentes facettes de l'activité professionnelle en question.

- Variation dénominative dans la sélection de la dimension: quand l'extension sélectionne différentes dimensions de classification du concept, ce qui donne une variation dénominative dans le modificateur du syntagme; par exemple, banc 
"marisqueiro » (accent sur l'activité de pêche qu'on y réalise) et banc naturel (accent sur l'origine naturelle du banc).

- Variation dénominative dans la sélection d'un trait appartenant à une même dimension: quand les unités reflètent la même dimension dans leurs extensions, mais moyennant un trait différent; par exemple, zone de production et zone de récolte, les deux extensions reflétant une activité fonctionnelle différente.

- Variation par l'inclusion/la suppression d'un trait: quand deux variantes sont différentes seulement par l'inclusion/absence d'un trait dans la base ou dans l'extension (cela se produit dans un même texte et l'explicitation des traits vient du fait que le locuteur considère qu'il est important de le faire dans certains contextes, mais pas nécessairement dans d'autres); par exemple, activité aquacole et activité économique aquacole.

- Variation dénominative dans le nombre de composantes pour expliquer la même information: quand les variantes offrent la même information sur le concept, mais diffèrent sur le plan du nombre de composantes utilisées; par exemple, concessionnaire et titulaire de concession ${ }^{3}$. (Fernández-Silva 2013: 31-33)

En ce qui concerne les raisons de ces variations, plusieurs recherches ont été réalisées autour de ce sujet, dont: Condamines et Rebeyrolle (1996) sur le point de vue, Péraldi (2012) sur l'indétermination des concepts et Tebé (2005) sur la variabilité de la structure conceptuelle d'une discipline. Toujours dans le cadre de la TCT, nous utiliserons dans cet article la proposition de Tebé (2005: 73), qui fournit une liste de facteurs ayant des conséquences sur la variation dans les productions communicatives d'un domaine:

- le stade de développement d'une science ou d'une technique;

- les différentes approches selon les écoles ou les courants de pensée;

- les visions du monde selon la culture;

- les interprétations divergentes de la réalité;

- les différents axes d'intersection dus aux interdisciplines;

- les intérêts des collectifs professionnels.

Notre objectif est ici d'analyser, à partir de ces typologies, la variation dénominative (avec conséquences cognitives) dans le domaine de la muséologie et de la muséographie dans un corpus composé de textes en espagnol.

\section{Corpus et méthodologie}

Les domaines du corpus d'étude sont la muséographie et le dessin d'exposition: secteur socioprofessionnel divers et pluriel, discipline pratique et d'origine théorique récente. Le corpus auquel nous faisons ici appel est tiré d'un corpus plus vaste (trilingue français/espagnol/catalan) bâti dans le cadre du projet de recherche FAR1 2016 (Dynamicité et variation dans le discours spécialisé du domaine de la muséographie). Aux fins du présent article, nous ne traitons que des données issues des textes espagnols, lesquels comptent au total 103050 mots.

Ce corpus de textes en espagnol est constitué de 18 textes écrits à l'origine en espagnol (il ne s'agit pas de traductions) par des experts en muséologie et en muséographie et qui traitent de la fonction exposition. Les textes sont notamment tirés de la revue Museos.es: Revista de la Subdirección General de Museos Estatales, d'autres revues spécialisées du domaine et d'une thèse doctorale ${ }^{4}$. Les textes ont été publiés entre 2006 et 2015. 
L'extraction terminologique automatisée du contenu des textes du corpus a été réalisée avec l'outil TermoStat ${ }^{5}$ de l'Observatoire de linguistique Sens-Texte (OLST) de l'Université de Montréal (Drouin 2003). Il s'agit d'un outil d'acquisition automatique de termes qui exploite une méthode de mise en opposition de corpus spécialisés et non spécialisés en vue de l'identification des termes. TermoStat reçoit un texte en entrée et retourne comme résultat principal une liste de candidats termes (CT) tirés du texte (site TermoStat, OLST). Dans le cas de l'espagnol, le corpus non spécialisé qui sert de point de comparaison à l'outil est issu de l'Assemblée parlementaire européenne et compte environ 30000000 d'occurrences (approximativement 527000 formes différentes).

TermoStat applique un filtre, à l'aide d'expressions régulières, qui est capable de nous donner une liste de candidats termes ordonnés par fréquence brute dans le corpus et par pondération (score), du plus pertinent au moins pertinent dans le texte ${ }^{6}$. Les pondérations peuvent être obtenues à partir de quatre tests différents: spécificité, $\mathrm{Chi}^{2}$, Log-likelihood et Log-odds ratio.

Dans le cadre de la présente étude, nous avons réalisé une extraction terminologique en tenant compte de la spécificité (pondération offerte par TermoStat). À partir des résultats obtenus, nous n’avons tenu compte que des unités ou des groupes d'unités comprenant le morphème muse(o)- afin d'obtenir les expressions incluant le mot museo et celles composées de museo- (comme museografía), museí- (comme

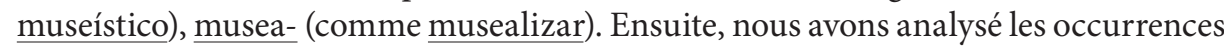
desdits candidats termes sélectionnés à partir de la fonction KWIC de TermoStat.

\section{Description : analyse quantitative et qualitative des données}

L'extraction terminologique des expressions incluant le morphème muse(o)- dans le corpus en espagnol a donné 466 candidats de regroupement à partir d'un total de 5429 candidats termes détectés. Parmi ces 466 candidats, plusieurs unités sont récurrentes dans l'usage et fonctionnent dans plusieurs groupes ou expressions complexes ${ }^{7}$ L'unité museo (musée) apparaît 1158 fois dans le corpus, y compris quelques occurrences dans un groupe nominal (dont 6 occurrences avec préfixe). Dans le cas des groupes nominaux, nous trouvons des structures où museo est le noyau du groupe nominal et où il est accompagné par un adjectif ou un complément de nom introduit par une préposition (notamment de), par exemple museo nacional (musée national) ou museo de arte (musée d'art). Nous avons également détecté quelques occurrences où museo est accompagné par un nom (museo casa; musée maison). Finalement, 27 occurrences présentent la structure de groupe nominal, dans laquelle museo est le complément du noyau et est introduit par une préposition (red de museos, montaje de museo; réseau de musées, montage de musée, respectivement).

La construction de groupe nominal avec l'adjectif museístico (littéralement muséistique) est présente dans 48 occurrences, alors que la construction avec l'adjectif museal (muséal) compte 14 occurrences. Toujours pour museal, notons la présence (bien que peu fréquente) de constructions liées à l'évènement musealizar (muséaliser; 1 occurrence), musealizado (muséalisé, accompagnant un nom; 3 occurrences) et musealización (muséalisation; 4 occurrences au total).

Quant à museología (muséologie), la plupart des phrases présentent cette unité seule (21 occurrences), quoique nous ayons relevé quelques utilisations dans un 
groupe nominal (avec adjectif ou avec complément de nom; 1 occurrence) ou avec l'utilisation du préfixe ciber- (cyber-; 2 occurrences).

En ce qui concerne museografía (muséographie), la fréquence de cette unité est beaucoup plus importante que museología (muséologie; 94 occurrences). Son utilisation avec le préfixe ciber- (cyber-) y est également plus élevée (21 occurrences), et l'utilisation de museografía dans un groupe nominal y est aussi plus importante, notamment lorsqu'il est question de combiner cette unité avec un adjectif (40 occurrences).

Enfin, en parallèle avec museología et museografía, les noms museólogo (muséologue) et museógrafo (muséographique) apparaissent dans le corpus, même s'ils sont rarement utilisés (un total de 9 occurrences entre les deux). Les adjectifs museológico (muséologique) et museográfico (muséographique), par contre, sont fréquents et apparaissent toujours dans une construction de groupe nominal d'un nom suivi de l'adjectif museológico (46 occurrences) ou de museográfico (105 occurrences).

Ainsi, museo fonctionne notamment tout seul ou comme noyau d'un groupe nominal. Quant aux formes adjectivales, celles dérivées des disciplines théorique et appliquée sont plus fréquentes dans le corpus (museológico et museográfico), même si les formes dérivées directement de museo produisent des dérivés d'autres catégories grammaticales (le verbe musealizar, l'adjectif ou le participe passé musealizado).

Ci-dessous, nous décrivons et analysons qualitativement et de façon plus détaillée le fonctionnement des unités détectées que nous venons de présenter de façon quantitative. Notre analyse est divisée en trois sous-sections bâties autour de trois termes productifs dans le corpus: $\underline{\text { museo, museología et museografía. }}$

\section{1. $\underline{\text { museo }}$}

La plupart des occurrences de museo apparaissent seules. Dans ces cas, le sens exprimé est simplement celui d'«établissement» ou d'«institution» (1):

(1) Nos referimos a la realización de importantes depósitos en otros museos que afectaron a sus fondos tanto cuantitativa como, sobre todo, cualitativamente, al permitir la salida de las piezas más destacadas.

Quelques occurrences présentent une variation dénominative moyennant un préfixe, soit webmuseo (2), soit cibermuseo (3). Dans ces cas concrets, les termes réfèrent à un type spécifique de musée:

(2) Dentro de estos museos online habría que dividir entre los que tienen un referente real (sin que implique que sea un calco de este), que serían los cibermuseos o webmuseos, y los que solo tienen existencia en la red, que serían los museos virtuales.

(3) Para hacer más operativa la clasificación, podría concluirse que los cibermuseos reproducen en el ciberespacio los tres elementos que los constituyen en su versión presencial (institución, edificio y colección), mientras que los museos virtuales virtualizan estos tres elementos, inventando un nombre para el museo, recreando un espacio tridimensional y creando una colección formada por obras pertenecientes a diferentes museos y colecciones.

Nous avons vu que l'unité museo apparaît à l'occasion accompagnée d'un adjectif, d'un complément introduit par une préposition et, de façon moins fréquente, d'un autre nom. 
Les combinaisons [museo + Adjectif] servent à exprimer le type d'administration de l'institution (4) ou sa portée (5), sa thématique (6) (7), ou des caractéristiques concernant la perspective ou l'infrastructure (8) (9) conçues pour ledit musée:

(4) Un montaje novedoso que nació apoyado por la creación de un museo público, en el que como decimos, se dio entrada a obras tan distintas como eran las comprendidas entre la pintura del final de la Edad Media y la del pintor Vicente López.

(5) Con algo de retraso con respecto a los museos europeos y americanos, los Departamentos de Educación y Acción Cultural, también llamados D. E. A. C., proliferaron en los museos españoles ante la demanda de nuevos servicios por parte del público escolar, ayudando a redefinir el papel del Museo609.

(6) En primer lugar, los museos arqueológicos, aunque con especiales connotaciones de valor histórico y documental, la propia Arqueología define a las piezas arqueológicas como artísticas, por lo que forman parte sin duda de una de las categorías de museos de arte.

(7) El Institut puso en marcha la recolección de fondos y colecciones que dieran testimonio material de la importancia que las actividades marítimas habían tenido en la sociedad y la cultura catalanas, y que constituyeran el núcleo primigenio de un museo marítimo.

(8) En segundo lugar, según el autor el otro tipo de museos de estilos, son los denominados museos ambientales que los integran aquellos que reconstruyen cuadros de época, adaptando el espacio arquitectónico al contenido museístico, los museos que incluyen reconstrucciones arquitectónicas y los de atmósfera de época que pretenden contextualizar la obra que albergan, como por ejemplo las secciones de esculturas clásicas de los museos europeos.

(9) Las disciplinas que estudian los museos virtuales se pueden denominar, al igual que en el caso de los museos presenciales, cibermuseología y cibermuseografía, siendo la primera la que se orienta hacia la reflexión teórica acerca de los museos online y la segunda la que analiza su plasmación en una página web.

Quant aux combinaisons [museo + Syntagme prépositionnel], elles servent à exprimer les mêmes types de renseignements que la structure précédente: type d'administration de l'institution (10), thématique (11) et caractéristiques concernant la perspective ou l'infrastructure (12) conçues pour ledit musée. Dans la plupart des cas, la préposition utilisée est de. Toutefois, l'usage de en apparaît dans les formes

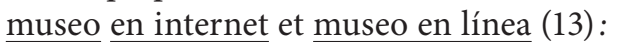

(10) Esta categoría la integran, en primer lugar, todos aquellos museos monográficos que corresponden a un estilo artístico concreto, como el museo del Romanticismo; los museos dedicados a la vida y obra de artistas como el Museo Picasso o el Museo de Van Gogh y los museos de coleccionista privados, de gran relevancia para nuestra investigación; pues uno de ellos, el Museu Deu, se convierte en el espacio de aplicación del estudio.

(11) Entre los museos de Bellas Artes se encuentran los grandes museos nacionales de arte surgidos a través de las grandes colecciones reales, de las desamortizaciones religiosas y legados, como la National Gallery, el Museo del Louvre o el Museo del Prado.

(12) El MArAs puedeconsiderarse la institución más relevante en lo que respecta al patrimonio arqueológico del territorio asturiano y ejerce un papel referencial para los museos de sitio, los yacimientos musealizados y los centros de interpretación de carácter arqueológico diseminados por el Principado. 
(13) Estos espacios web creados por los museos hanser calificados indistintamente como museo virtual, museo online, museo en línea, webmuseo, museo digital, cibermuseo, o, en definiciones más complejas, centro virtual de interpretación, egalab (electronic galleries and laboratories) o plataforma virtual museística.

Les constructions [museo + Nom] se réduisent à quelques unités terminologiques très précises qui réfèrent notamment à un type d'infrastructure muséale: une maison qui sert de musée et qui est elle-même considérée comme un élément du parcours et des discours muséaux (dans le cas de notre corpus, les deux occurrences de ce type réfèrent, en fait, à des noms propres: Museo Casa de Cervantes, par exemple). Nous trouvons également une occurrence avec la même structure qui réfère à un concept de l'histoire de la muséologie, les musées objets (14):

(14) No suelen formar parte de estos museos objetos posteriores al siglo XV.

Il existe également des combinaisons où museo est le deuxième nom dans le groupe syntagmatique. Dans ces cas, museo accompagne vivienda ou ciudad (15):

(15) Veinte años más tarde, cuando el museo ha salido de las paredes, cuando hablamos de ciudades museo y los destinos turísticos se rigen por puntos de interés tratados museográficamente, he tenido la suerte de ser solicitado para desarrollar estos nuevos productos turísticos de carácter museístico.

Enfin, le corpus présente plusieurs combinaisons syntagmatiques qui prennent

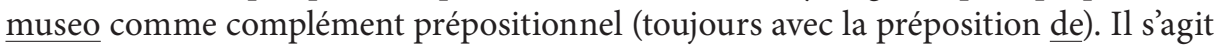
d'expressions d'actions muséologiques ou muséographiques dont le récipiendaire est le musée et l'unité noyau du syntagme d'un nom déverbal (16) (17):

(16) Sin embargo, la salida de estas piezas, incluidas la magnífica colección de lápidas hebreas, no fue tan definitiva como los cuadros del Greco, al volver a formar parte del montaje del Museo Arqueológico provincial inaugurado en 1931. Su segregación definitiva se produjo en época mucho más reciente con motivo de la inauguración del Museo Sefardí en la Sinagoga del Tránsito el año@ord@ Al final, el desarrollo del turismo en la ciudad de Toledo ligado en buena medida a la recuperación de la figura del Greco, hizo posible el inicio de la restauración del antiguo convento de San Juan de los Reyes.

(17) En 1930, y como continuidad a la política restauradora de grandes edificios monumentales emprendida en Toledo a comienzos del siglo xx, le llegó el turno a Santa Cruz y muy poco después, en 1935, se pudo realizar el montaje del museo en unas nuevas dependencias más amplias de las que había dispuesto hasta entonces. (Aragoneses 1957: 26)

Dans le corpus, on utilise également cette structure pour indiquer des professions liées à la pratique muséale: directoras de museo et personal de museo.

Les dérivations adjectivales museístico et museal sont également productives, que ce soit pour compléter des unités exprimant des actions (18), des objets (19) ou des installations (20) et des concepts abstraits (21):

(18) Los programas de investigación museográfica Con base en los estudios registrados quedan establecidos cuatro programas de investigación museográfica que contribuyen a la comprensión del fenómeno pedagógico museal, ellas son: investigaciones centradas en lo museográfico, investigaciones centradas en el comportamiento museal, investigaciones centradas en las interacciones público-exhibición y investigaciones evaluadoras. 
(19) Ellos encuentran comportamientos muy diferentes en el medio museal, y afirman que es posible identificar comportamientos de aprendizaje diferentes entre los dos tipos de visita, por ejemplo, reportan que las personas solas aparentan leer más que las personas acompañadas, y que las acompañadas pasan más tiempo con los objetos museales que las primeras.

(20) El evento es lo emergente, es plasticidad puesto que es una síntesis del proceso que se da del encuentro entre los visitantes y el espacio museal, lo cual ocurre a cada instante y no se puede prefigurar o predecir.

(21) Estas ideas hanser recogidas por varios autores en los últimos años y se ejemplifican con la sentencia de Weil (1999) sobre el cambio en el modelo museístico de "being about something to being for somebody".

L’adjectif museístico apparaît fréquemment dans le corpus comme complément de l'unité institución (17 occurrences), ce qui donne institución museística comme synonyme de museo. Nous avons donc trouvé intéressant de regarder les autres occurrences où le terme institución comme synonyme de museo est utilisé dans ce même corpus: institución (22), institución cultural (23), institución patrimonial (24).

(22) Con él se pudo mostrar al público una parte significativa de los fondos atesorados, dando lugar a un cambio en la visión del centro por parte de los responsables políticos que se comprometieron con el futuro de la institución.

(23) Pero más allá del tipo de fondos, los museos de Arte Contemporáneo se enfrentan a ciertos retos para mantener un equilibrio entre su esencia y su función como institución cultural.

(24) Además estas formas de curaduría digital están ampliamente implementadas en museos y otras instituciones patrimoniales desde la segunda mitad del siglo XX. (Parry y Sawyer, 2005)

De façon similaire, les unités avec centro sont productives en tant que synonyme de museo. Dans le corpus, nous trouvons notamment: centro (25) et centro de interpretación (26).

(25) Un hecho que podemos comprobar con un breve análisis de la oferta de los museos públicos de Toledo que, si a finales de los años setenta constaba de ocho centros, en la actualidad y tras algunas esperadas aperturas y reaperturas, se ha reducido a cinco, de los que solo tres, el Sefardí, el dedicado al Greco o el del Ejército, se encuentran a pleno rendimiento.

(26) Si bien el carácter y los objetivos de los centros de interpretación difieren de aquellos que son propios de los museos, en todas estas instalaciones encontramos una vocación didáctica común que guía tanto sus nuevos discursos expositivos, como sus recursos museográficos.

Enfin, la version verbale musealizar, ainsi que son participe passé utilisé comme adjectif (musealizado) et le nom déverbal qui en dérive (musealización) sont également présents dans le corpus pour exprimer des actions et des résultats (27) (28) (29):

(27) Los museos de arte y sus categorías desde la teoría museológica Una de las primeras cuestiones a tener en cuenta es la gran cantidad y diversidad de museos de arte que hay en todo el mundo porque el arte es un fenómeno universal y todas las culturas conocidas poseen manifestaciones artísticas, y además, casi todas las sociedades han llegado al proceso intelectual-cultural de musealizar, bien las obras de arte de su propia cultura, como las de otras. 
(28) Más allá de estos acentos de la colección, el MArAs puedeconsiderarse la institución más relevante en lo que respecta al patrimonio arqueológico del territorio asturiano y ejerce un papel referencial para los museos de sitio, los yacimientos musealizados y los centros de interpretación de carácter arqueológico diseminados por el Principado.

(29) Su función puede llegar a ser tan básica como la de otros grandes complejos urbanos al depender de ellos la correcta y necesaria musealización del territorio.

\section{2. museología}

Dans la plupart des occurrences de museología, l'unité apparaît toute seule, c'est-àdire qu'elle n'a pas de compléments et qu'elle ne fonctionne pas non plus comme complément d'autres unités (30):

(30) Para dicha publicación se ha contado con las reflexiones de diferentes profesionales de la museología y de los estudios feministas.

Il importe de mentionner également la combinaison [Préfixe + museología] qui donne la forme cibermuseología (31) et les structures avec museología comme complément d'un nom et avec la préposition de. Dans ces derniers cas, on exprime des ressources en lien avec l'étude des musées (32):

(31) Las disciplinas que estudian los museos virtuales se pueden denominar, al igual que en el caso de los museos presenciales, cibermuseología y cibermuseografía, siendo la primera la que se orienta hacia la reflexión teórica acerca de los museos online y la segunda la que analiza su plasmación en una página web.

(32) En revistas de museos organizadas por universidades que ofrecen planes de estudios a nivel de posgrado como el caso de la Universidad de Leicester en Inglaterra que publica la revista Museum and Society, una de las más reconocidas, y la Universidad Complutense de Madrid, que tiene la Revista de Museología.

La combinaison la plus productive reste cependant le syntagme [Nom + museológico] ; elle est donc dérivée de museología et utilisée pour exprimer des concepts (33) (34) et des installations muséales (35):

(33) Así, el modelo museológico dominante se materializa a través de un discurso estructurado desde el museo y desde sus departamentos técnicos enfocados en el consumo de los usuarios.

(34) Por otra parte, se eligió Reino Unido como " contraparte " del estudio ya que representa la tradición museológica anglosajona, completamente diferente a la tradición mediterránea que pone el énfasis en la difusión, la comunicación y la educación como fines de la función museológica. Esto ha llevado a que Reino Unido sea uno de los pioneros en el desarrollo de estrategias y recursos interactivos en todas las categorías de museos, incluidos los museos de arte.

(35) En Grecia es donde surgen los primeros espacios museológicos fruto de la religiosidad popular, ya que las primeras obras de arte coleccionadas eran exvotos de los fieles que se guardaban en los recintos sagrados.

Enfin, plusieurs occurrences expriment la profession de muséologue (36):

(36) Es decir, que generalmente la museografía de los museos de arte, hoy en día sigue siendo convencional y los museólogos más académicos la siguen defendiendo como la única concepción válida. 


\section{3. museografía}

La description des occurrences de museografía et de ses dérivés reste assez parallèle à celle dont nous avons fait état pour museología. En effet, la plupart des occurrences de museografía ne présentent pas l'unité avec compléments ni ne fonctionnent comme complément d'autres unités, mais seules (37):

(37) También el mundo de las mentalidades de los grupos humanos del Paleolítico tiene un lugar primordial en el discurso que se refleja a su vez en la museografía: para realzar las piezas de arte mueble se emplea una iluminación de acento con leds y un buen número de ellas se exhiben a través de una vitrina de doble visión que permite enriquecer la contemplación de estas magníficas piezas desde varios puntos de vista.

Mentionnons également la combinaison [Préfixe + museografía] qui donne la forme cibermuseografía (38) et les structures [museografía + Adjectif]. Dans ces derniers cas, on exprime des ressources (39) et des concepts ou des installations en lien avec l'étude de la pratique muséographique (40):

(38) La definición de los modelos didácticos en el ámbito de la cibermuseografía se ha realizado mediante tres estudios de caso: el Museo del Prado como ejemplo de página web educativa tradicional-tecnológica, que constaba de 8 actividades didácticas interactivas en su página web PradoMedia, el Centro Andaluz de Arte Contemporáneo como activista-espontaneísta, que ha desarrollado Aprendiendo con la colección, y el National Museums Online Learning Proyect como referente innovador-investigativo, un proyecto conjunto entre nueve museos británicos, que se ha plasmado en la elaboración de 108 webquests en las que relacionan las colecciones de estos museos con el currículo nacional británico.

(39) Otros recursos museográficos complementarios (maquetas, audiovisuales e interactivos) amplían o apoyan el contenido del discurso con el fin de favorecer su comprensión.

(40) Uno de los retos a los que se enfrenta la propuesta museográfica es la ya citada relación con la luz natural en una ciudad como Oviedo, que presenta un bajo índice de horas de sol y luz por año. (1.711h)

Cependant, à la différence des cas de museología, la combinaison de museografía avec un complément (notamment avec un adjectif, mais aussi avec un complément introduit par la préposition de) est productive dans le corpus étudié (41) (42):

(41) La museografía interactiva, sin duda, debería estar presente en los museos de arte porque su empleo podría hacer más accesibles los contenidos de los museos a un mayor público, lo cual ya es propio de otras tipologías de museos como los de Ciencias o los de Historia.

(42) Una vez que se conozcan, la nueva museografía del arte tiene que crear recursos basados en estos mecanismos del placer para que sean capaces de generarlo en la contemplación del arte por parte del espectador.

La combinaison la plus productive reste toutefois le syntagme [Nom + museográfico] ; elle est donc dérivée de museografía et utilisée pour exprimer des concepts (43) et des résultats ou pour nommer des types d'installations d'exposition (44) (45):

(43) Así, la pedagogía museográfica no está claramente sistematizada y son los resultados de investigaciones en este campo los que nos pueden dar una luz que permita una propuesta en este sentido. 
(44) En 2009 el Ministerio de Cultura convocó el concurso público para la redacción y ejecución del proyecto museográfico que fue adjudicado en diciembre de dicho año a la unión temporal de empresas Jesús Moreno y Asociados.

(45) Una experiencia vital con dos protagonistas, el destino y el visitante, entre los que se ha de desarrollar una puesta en escena del destino para que el discurso y diseño museográfico sean de interés y accesibles a todos los visitantes.

Enfin, notons la présence de plusieurs occurrences exprimant la profession de muséographe (46):

(46) Esos micro espacios cuentan con objetos virtuales, en tanto que fueron diseñados para ser parte de una puesta en escena educativa con unos significados concretos desde el punto de vista del museógrafo, pero los visitantes co-interpretan tales objetos desde múltiples perspectivas y referentes.

Avant de considérer dans notre corpus les variations dénominatives avec conséquences cognitives à partir de la typologie de Fernández-Silva (2013), il faut mentionner l'existence de variations dénominatives sans conséquences cognitives. Cela signifie qu'il n'y aurait pas de variation sémantique, seulement une variation formelle: museo presencial et museo de forma presencial, museo arqueológico et museo de arqueología, ou recurso de museografía et recurso museográfico, entre autres.

Ainsi donc, en ce qui concerne notre corpus, et pour les unités extraites autour de muse(o)-, il est possible de déceler notamment deux types de variations récurrentes du point de vue exclusivement formel à partir de la classification proposée par Freixa (2002: 281-282) que nous avons vue ci-dessus:

- Changements morphosyntaxiques avec changement de structure: par exemple museo arqueológico et museo de arqueología.

- Changements lexicaux: de base (par exemple, centro museístico et institución museística) ou d'extension (par exemple, espacio museístico et espacio museal).

\section{Analyse de la variation dénominative}

Dans cette section, nous considérons plus en détail les types de variations à partir de la classification de Fernández-Silva (2013 : 31-33), c'est-à-dire notamment des variations avec des conséquences cognitives.

\subsection{Types de variations dénominatives dans le corpus}

Les formes red de museos (réseau de musées) et asociación de museos (association de musées) dans notre corpus constituent un exemple de variation dénominative dans la sélection de la catégorie. Quand le locuteur parle de red, l'ensemble des musées concernés est conceptualisé comme étant situé dans une zone géographique ou thématique précise et échangeant des ressources de différents types. Quand il s'agit d'asociación, l'ensemble des musées est conceptualisé comme un regroupement sans autres spécifications.

Les occurrences des termes museografía accesible et museografía accesible e inclusiva constituent, pour leur part, un exemple de variation dénominative avec l'ajout d'un trait qui indique le caractère inclusif de la muséographie en question.

Les exemples de variation dénominative présents dans le corpus et qui réfèrent à des éléments événementiels illustrent une variation sur le plan de la sélection de la 
catégorie. Le choix terminologique fait par le locuteur reflète donc sa façon d'aborder le domaine muséal et l'évènement en question: inauguración/apertura del museo, instalación/montaje del museo, ainsi que diseño/disposición/instalación museográfica.

Dans le premier cas, inauguración permet de conceptualiser l'évènement comme une fête ou une réception, tandis qu'apertura (ouverture) est conceptualisée comme un simple évènement d'ouverture au public. Quant à instalación et montaje, les occurrences indiquent une tendance, dans le cas d'instalación, à conceptualiser le résultat de la réalisation muséale, tandis que montaje permet de conceptualiser de façon plus marquée le processus et non le seul résultat. Finalement, pour le groupe diseño, disposición et instalación, plusieurs perspectives de conceptualisation seraient mises en exergue lors de l'utilisation de chacun des termes en question: le

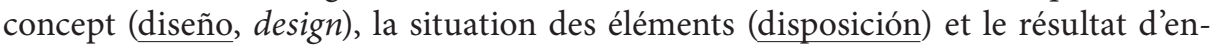
semble (instalación).

Des unités telles que museo casa (musée maison) et vivienda museo (logement musée) constituent des exemples de changement de configuration conceptuelle (y compris la base, l'extension et la relation intraconceptuelle), ce qui a des conséquences cognitives très importantes pour la compréhension des unités terminologiques en question. En effet, l'ordre syntaxique [Base + Extension] est essentiel au moment de déceler le sens de chacune de ces dénominations: museo casa fait référence à un musée qui autrefois était la résidence d'un personnage célèbre réel ou fictif (47), tandis que vivienda museo renvoie à un logement qui sert également de musée (48):

(47) Dentro de esta política, que al menos sirvió para asegurar la integridad de las colecciones que formaban parte del antiguo Museo Provincial de Toledo, hay que situar la inauguración del Museo Taller del Moro en el año 1963 (Revuelta 1979), del Museo Ruiz de Luna en Talavera de la Reina ese mismo año (Manso 2008), del Museo Casa de Dulcinea del Toboso en esa localidad en 1967 (Revuelta 1973b), del Museo de los Concilios y de la Cultura Visigoda y del Palacio de Fuensalida en 1971 (Revuelta 1973a) y, por último, del Museo de Arte Contemporáneo en la Casa de las Cadenas en 1975. (Puente y Santa Ana 1975)

(48) La informatización de los habitantes nos ha llevado a respetar la vivienda museo, intervenir solamente para hacerla accesible e inclusiva y utilizar las nuevas tecnologías audiovisuales, en su interior, para complementar el discurso de la visita y aplicar el Street Museum10 en el exterior, para acercarnos al contexto de lo que fue la cotidianidad del pueblo de Masquefa en el siglo pasado.

Nous pouvons également grouper des unités terminologiques en fonction du fait qu'elles présentent des extensions qui reflètent une même dimension (caractéristique immersive de l'exposition), mais au moyen d'un trait différent: museo ambiental/ $\underline{\text { museo de ambiente }}^{8}$ (le public se trouve dans un environnement qui reproduit une époque ou un lieu), museo de estilo (le public se trouve entouré par le style d'une époque), museo de exhibiciones contextuales (l'objectif des expositions est de permettre au public de faire partie de l'environnement du lieu ou de l'époque présenté), museo de sitio (l'environnement recréé dans les salles du musée fait en sorte que le public a la sensation d'être à l'époque ou dans les lieux présentés).

Le corpus étudié présente des exemples de variation dénominative dans le changement de la dimension ou d'un trait de la dimension. Il s'agit de museo virtual, museo en internet, $\underline{\text { museo online/museo en línea }}{ }^{9}$, webmuseo, cibermuseo. Toutefois, la façon d'utiliser ces unités dans le corpus exige quelques remarques supplémentaires. 
En effet, selon les besoins discursifs, le locuteur peut utiliser ces unités en tout ou en partie pour se référer à l'idée d'un musée non présentiel, ce qui indique qu'il s'agit de variantes dénominatives (49):

(49) Estos espacios web creados por los museos son calificados indistintamente como museo virtual, museo online, museo en línea, webmuseo, museo digital, cibermuseo, o, en definiciones más complejas, centro virtual de interpretación, egalab (electronic galleries and laboratories) o plataforma virtual museística.

Cependant, lorsqu'un besoin discursif exige des distinctions spécialisées (par exemple, une nécessité pédagogique pour des apprentis muséologues ou muséographes), des nuances sont faites sur le plan des distinctions sémantiques de ces unités terminologiques. Ainsi, la distinction conceptuelle fait en sorte que certaines de ces unités ne sont pas utilisées comme variantes dénominatives dans d'autres contextes détectés dans le corpus.

Ainsi, museo online, museo en línea et museo en internet expriment cette idée du non-présentiel sans d'autres spécifications. Il y a toutefois une claire distinction parmi les experts entre cibermuseo et webmuseo (des musées en ligne qui sont en lien avec un musée présentiel existant) et museo virtual (musée exclusivement en ligne) (50):

(50) Dentro de estos museos online habría que dividir entre los que tienen un referente real (sin que implique que sea un calco de este), que serían los cibermuseos o webmuseos, y los que solo tienen existencia en la red, que serían los museos virtuales.

Des occurrences comme celles de cibermuseo et de webmuseo détectées dans le corpus nous montrent que les variantes dénominatives ne sont pas exclusivement constituées d'un adjectif ou d'un complément du nom, mais qu'il peut aussi s'agir de l'ajout d'un affixe dérivationnel (ciber- ou web-, dans le cas de notre corpus). Il s'agit d'une variante que Freixa (2002) classifie comme changement morphosyntaxique modifiant la structure. Ainsi, nous pourrions ajouter une spécification dans la classification de Fernández-Silva (2013), par laquelle la variation dénominative s'adapte à d'autres processus de formation de termes non syntagmatiques. Dans ces cas-ci, cette variation aurait lieu sur le plan de l'abstraction de la catégorie et créerait des liens d'hyperonymie (museo) et d'hyponymie (cibermuseo, webmuseo, etc.).

Enfin, il est également intéressant de considérer le groupe d'unités terminologiques suivant: museografía didáctica, cibermuseografía didáctica, museografía interactiva, pedagogía museográfica, didáctica museográfica. La variation dénominative est présente dans la sélection de la dimension entre les unités museografía didáctica et museografía interactiva: dans le premier cas, le locuteur explicite la volonté d'apprentissage de ce type de muséographie tandis que dans le deuxième cas, il met en exergue l'aspect interactif de l'approche.

Quant à la variation de cibermuseografía didáctica, elle semble répondre à un phénomène similaire à celui que nous avons exposé entre museo et cibermuseo: on met en exergue certaines facettes de la catégorie (ici, une activité) moyennant une variation dénominative sur le plan de l'abstraction de la catégorie (la classe conceptuelle reste la même, mais on se trouve dans des niveaux d'abstraction différents). Les unités museo ou museografía didáctica incorporent donc une spécification avec l'ajout d'un préfixe, ce qui crée un lien d'hyperonymie/hyponymie avec les unités incluant le préfixe (respectivement cibermuseo et cibermuseografía didáctica). 
La variation entre pedagogía museográfica et didáctica museográfica semble se situer dans la sélection de la catégorie de la base. En effet, même si les unités pedagogía et didáctica sont utilisées souvent comme synonymes dans les échanges non spécialisés, les textes du domaine de l'éducation s'efforcent souvent de faire la distinction suivante: pedagogía reflète la transmission de contenus moyennant la relation maître-élève (ici relation musée-visiteur), tandis que didáctica reflète la transmission de ces contenus moyennant la façon et les méthodes de présenter le contenu pour qu'il soit accessible et compréhensible (ici discours muséographique). Finalement, la principale différence entre les deux groupes de termes, selon que leur base est museografía ou pedagogía/didáctica, réside dans la configuration conceptuelle et fort probablement dans les acteurs professionnels qui s'y réfèrent: des muséologues ou des enseignants.

\subsection{Patrons récurrents de variation dénominative dans le corpus}

Dans le cadre de notre recherche, la plupart des variations dénominatives détectées ne présentent pas seulement un des types de variations proposés par Fernández-Silva (2013), mais des patrons récurrents. Également, il est possible d'établir un lien entre ces variations récurrentes et les facteurs de variabilité de Tebé (2005) vus ci-dessus.

Le premier élément récurrent de ces variations dénominatives est lié à la variation dénominative qui se produit entre museología et museografía (variation dans la sélection de la catégorie, c'est-à-dire que différentes conceptualisations de la discipline muséale sont mises en exergue; respectivement 21 et 94 occurrences). En effet, pour se référer à quelque chose lié aux musées ou à leur discipline, le locuteur utilise plusieurs options d'extension: de museo, museístico et museal (de museo), museológico (de museología) et museográfico (de museografía).

Dans le cas de museístico et museal (avec respectivement 42 et 14 occurrences; adjectif fonctionnant comme complément nominal), il semble s'agir d'une variation dénominative sans conséquences cognitives qui n'entraîne qu'un changement morphologique (par exemple, espacio museístico et espacio museal). Le choix de l'un ou de l'autre complément ne semble pas comporter de conséquence sémantique ou conceptuelle sur le terme en question (l'usage de museal semblerait avoir son origine simplement dans la tradition de textes français du domaine). Toutefois, en ce qui concerne de museo (27 occurrences), le changement syntaxique ([Nom + Adjectif] ou [Nom + Syntagme prépositionnel]) semble avoir des conséquences cognitives. En effet, les occurrences avec de museo semblent référer aux musées de façon générale (appartenance: objeto de museo, red de museos, etc.), tandis que les versions avec les adjectifs (objeto museístico, objeto museal) activent un trait de réflexion disciplinaire autour de l'unité centrale qui n'est pas nécessairement incluse dans la combinaison avec complément introduit par une préposition. Les différents axes d'intersection dus aux interdisciplines, ainsi que les intérêts des collectifs professionnels qui peuvent en dériver (deux facteurs de variabilité proposés par Tebé 2005), et qui établissent ici les différents emplois dans un musée, pourraient favoriser l'existence de cette variation récurrente (différents points de vue du conservateur des collections, du technicien en muséologie, du muséographe chargé de monter les expositions, etc.).

Dans le cas des trois compléments mentionnés ci-dessus (de museo, museístico et museal), de même que pour museológico (46 occurrences) et museográfico 
(105 occurrences), la variation dénominative se situe dans la sélection d'un trait appartenant à une même dimension: respectivement, le musée en tant que tel (trois options dans le corpus avec les nuances que nous venons de remarquer dans le paragraphe précédent), la discipline qui s’intéresse aux musées et la discipline appliquée qui a pour objet la fonction d'exposition des musées (par exemple, espacio/contexto/ objeto museístico/museal/de museo/museológico/museográfico).

Ainsi, à partir de ces combinaisons de paires dénominatives (de museo, museístico, museal; museología, museografía; museológico, museográfico, etc.), qui sont très productives dans le corpus de la discipline, les cas de variation dénominative se multiplient grandement, que ce soit par changements dans la base, dans l'extension ou dans les deux éléments du groupe syntagmatique.

À nouveau ici, les différents axes d'intersection dus aux interdisciplines, ainsi que les intérêts des collectifs professionnels qui peuvent en dériver (Tebé 2005) sembleraient favoriser ces patrons de variation récurrente.

Des unités comme espacio museístico/museal/, etc. et planta de museo, medio museal ou sala museográfica (dont la base et l'extension changent) semblent également liées par un changement dans la configuration conceptuelle. Quand le locuteur parle de planta de museo, l'espace muséal est conceptualisé d'un point de vue architectural en tenant compte des entrées, des sorties, etc. Quand il parle de medio museal, l'espace est conceptualisé comme le lieu où l'on peut placer des artéfacts. Quand le locuteur parle de sala museográfica, il conceptualise l'espace muséal en visualisant les éléments muséographiques qui ont été conçus pour l'exposition.

D’autres paires d'unités semblent liées par variation dénominative dans la sélection de la catégorie, telles que contenido museístico et colección de museo. En effet, quand le locuteur parle de contenido, l'ensemble d'artéfacts est conceptualisé comme des objets exposés dans le musée. Quand le locuteur parle de colección, l'ensemble d'artéfacts est conceptualisé comme un groupe d'objets ayant été recueillis et collectionnés par le musée en suivant une thématique, etc. En ce qui a trait à ces deux dernières unités, fondo museográfico et patrimonio museológico apparaissent dans le corpus en tant qu'expression d'un changement de configuration conceptuelle (base et extension). Dans le cas de leur base, il s'agit de variation dans la sélection de la catégorie. Quand le locuteur parle de fondo, l'ensemble est conceptualisé comme un groupe d'objets possédés administrativement et légalement par le musée. Quand le locuteur parle de patrimonio, l'ensemble d'artéfacts est conceptualisé comme une possession qui est l'héritage d'une collectivité. Les deux facteurs de variabilité de Tebé (2005) proposés ci-dessus sembleraient jouer un rôle également dans la variabilité détectée dans ces unités.

Il faut mentionner que notre corpus renferme d'autres exemples de changement dans la configuration conceptuelle (base et extension), dont: proyecto museológico, plan museológico, programa museológico, propuesta museográfica, proyecto museográfico, plan museográfico, etc.

Enfin, un autre groupe de termes intéressants en raison de leurs liens de variation dénominative détectés dans le corpus est composé de museo, institución, centro, institución museal et institución museística. Entre institución et institución museís$\underline{\text { tica, }}$ il semble n'y avoir que l'ajout d'un trait explicitant qu'il s'agit du domaine muséal; il s'agirait simplement de l'inclusion d'un trait additionnel comme le décrit Fernández-Silva (2013), où la variation serait sur le plan intratextuel. 
Toutefois, entre museo, institución (museal, museística) et centro, il y a de la variation dénominative dans la sélection de la catégorie, laquelle reflète différentes conceptualisations de l'entité musée: établissement officiel, endroit de rencontre ou consacré à un but spécifique (muséal, dans ce cas).

\section{Conclusions}

Notre analyse permet de dresser les constats ci-dessous en lien avec la typologie de Fernández-Silva (2013):

- cette typologie est applicable à d'autres domaines de spécialité;

- le concept d'« extension» peut être élargi de façon à inclure des préfixes ayant cette fonction (comme la proposition de Freixa 2002 le mentionnait); c'est-à-dire que la classification ne s'applique pas seulement aux termes composés, mais aussi à des termes créés par d'autres processus de formation (composition culte, composition patrimoniale, dérivation, etc.).

- l'ajout de préfixes semble se produire dans un type de variation concret, celui que Fernández-Silva (2013) situe sur le plan de l'abstraction de la catégorie et qui crée des relations d'hyperonymie et d'hyponymie.

En ce qui concerne la description terminologique du corpus d'un domaine, nous avons remarqué:

- que la variation dénominative dans des unités représentant des concepts noyaux de la discipline fait en sorte que le niveau de variation dans le corpus est très élevé, non seulement en ce qui a trait aux mots clés en question (museo, museología, museografía), mais aussi pour les unités créées à partir de ceux-ci (espacio museístico, espacio museológico, espacio museográfico);

- que ladite variation fait en sorte que certains patrons de variation dénominative deviennent récurrents, que ce soit dans la base (museología didáctica, museografía didáctica), dans l'extension (objeto museológico, objeto museográfico) ou dans les deux (proyecto museológico, propuesta museográfica).

Quant aux facteurs de cette variabilité, nous en avons vu deux parmi ceux proposés par Tebé (2005: 73) qui semblent être récurrents pour le domaine muséal au moment d'expliquer les patrons de variation détectés. Il s'agirait des différents axes d'intersection dus aux interdisciplines, ainsi que les différents intérêts des collectifs professionnels.

En effet, ces facteurs peuvent facilement s'appliquer au domaine avec lequel nous avons travaillé ici. Dans Joan Casademont et Lorente (2005, 2006a, 2006b), nous proposions une caractérisation de la discipline muséale qui indique certains de ces mêmes éléments, par exemple le fait que ce domaine d'étude est multidisciplinaire et donc qu'il fait usage de termes d'origines variées provenant de la conservation, de la restauration, de l'exposition, du droit et de l'économie, entre autres.

Également, il s'agit d'un domaine qui s'inscrit dans les sciences sociales et artistiques, lesquelles diffèrent des sciences nommées "exactes» (ces dernières ont tendance à favoriser la relation sans équivoque entre un terme et un concept, souvent par la standardisation, et présentent de nombreux termes éloignés de l'usage commun). De même, la discipline en question est d'origine pratique avec une éclosion théorique récente en comparaison d'autres disciplines scientifiques. Donc, la théorisation délimiterait quelquefois a posteriori des concepts souvent polysémiques, avec 
des dénominations variées et très proches des usages discursifs non spécialisés (Joan Casademont et Lorente 2005, 2006a, 2006b).

Enfin, il faut mentionner ici un aspect détecté lors de l'analyse et que nous trouvons très intéressant, puisqu'il sert à complémenter notre compréhension des facteurs liés à la variation dans le corpus étudié. En effet, dans plusieurs exemples du corpus, il est possible de voir que, sur le plan du discours, le locuteur a parfois le besoin de définir certains termes et de distinguer ceux qui vont être utilisés comme synonymes et sans aucune distinction ailleurs dans le corpus. Nous avons vu cela avec des termes tels que museo virtual, cibermuseo, etc., exemplifiés dans (49) et (50), ou avec la paire pedagogía museográfica et didáctica museográfica, commentée à la fin de la section 4.1.

Ainsi, indépendamment de la variabilité du domaine, il existe une variation liée au genre de communication ou au groupe de locuteurs considérés (experts avec experts, experts avec apprentis). Les facteurs situationnel et fonctionnel ne peuvent donc pas être oubliés. Les aspects liés au locuteur qui produit le discours sont importants (subjectivité), même s'il est également essentiel de considérer comment il envisage les locuteurs qui en seront les destinataires, leurs connaissances du sujet et comment ils risquent d'interpréter son discours (intersubjectivité).

Puisque le corpus que nous avons utilisé ici ne présente pas de distinctions précises en lien avec le genre de communications, nous pouvons seulement envisager de façon très générale des situations qui provoqueraient, sur le plan pragmatique, cette variation de distinction et de non-distinction entre unités terminologiques:

- Le locuteur qui produit le discours se situe en tant qu'expert enseignant et visualise le destinataire de son discours en tant qu'apprenti qui devra bien comprendre les nuances qui distinguent les différents termes du domaine en question.

- Le locuteur veut présenter une théorie et convaincre son interlocuteur de la pertinence de celle-ci. Toutefois, afin de pouvoir le faire, quelques distinctions claires entre les termes doivent être faites à certains points de son discours argumentatif.

Dans le cas de museo virtual, cibermuseo, etc., exemplifiés dans (49) et (50), la variation semble pouvoir s'expliquer avec la première des options données ci-dessus, puisque les occurrences du corpus proviennent d'un manuel de muséographie interactive (compte rendu). Quant à pedagogía museográfica et didáctica museográfica, commentés à la fin de la section 4.1., la variation pourrait s'expliquer par la deuxième des options présentées, puisque les occurrences concernées sont tirées d'articles scientifiques argumentatifs (une étude sur les visiteurs des musées et une étude sur les pages Web éducatives de musées virtuels d'art). La création d'un corpus avec des distinctions plus précises concernant les genres de communications serait une voie intéressante pour continuer la recherche sur ce sujet.

Nous avons vu que la variation dénominative met donc en relief les nuances cognitives des différentes formes avec lesquelles les locuteurs peuvent apprivoiser une réalité scientifique (Tercedor 2004: 199). La notion de point de vue définie notamment par Fernández-Silva (2013: 12) devient également ici un facteur essentiel, c'est-à-dire la position depuis laquelle un auteur traite d'un concept concret et qui a de l'influence sur la perception des traits distinctifs de ce concept. Aussi, la considération pragmatique du destinataire du discours semble jouer un rôle sur la variation.

Que ce soit en vertu des besoins du locuteur sur le plan du discours ou d'autres facteurs énumérés ci-dessus, l'étude de la variation dénominative est essentielle pour 
la bonne compréhension des textes de spécialité. Elle doit donc être intégrée dans les ouvrages terminologiques du domaine en question. Nous avons vu que plusieurs variations avaient des conséquences cognitives très importantes et donc bien les connaître est essentiel pour comprendre, entre autres, comment les experts conceptualisent leur discipline. De plus, le comportement récurrent de certaines combinaisons de variation dénominative que nous avons décrit permettrait, encore dans des ouvrages du domaine, de présenter les nuances cognitives de ces variations de façon succincte et efficace sans avoir à les répéter cas par cas.

Sans ce type d'analyse, les produits terminographiques comme les bases de données et les dictionnaires spécialisés ne peuvent offrir qu'un produit approximatif (quant au type d'information et au détail de l'information) à l'intention des experts ou des traducteurs. Ceci peut poser problème non seulement sur le plan de la communication et de la diffusion d'information entre experts, mais aussi avec les non-initiés.

\section{REMERCIEMENTS}

Cette recherche a été réalisée dans le cadre du projet FAR1: Dynamicité et variation dans le discours spécialisé du domaine de la muséographie (Université TÉLUQ). La collecte de données n’aurait pas été possible sans la collaboration du Centre de documentation et de recherche du Musée du Jouet de Catalogne à Figueres [www.mjc.cat]. Enfin, je remercie sincèrement Sabela Fernández-Silva pour ses commentaires et suggestions sur le contenu, ainsi que Jacqueline Chevalier et Marc Pomerleau pour la révision.

\section{NOTES}

1. Variation dénominative: «phénomène par lequel un concept spécialisé est dénominé par diverses unités terminologiques distinctes, lesquelles peuvent différer formellement, mais aussi sémantiquement» (notre traduction).

2. L'unité marisqueiro signifie «relatif aux coquillages».

3. Notre adaptation de l'espagnol.

4. Pour de plus amples détails sur les documents formant le corpus en espagnol, consultez l'Annexe 1.

5. Drouin, Patrick (2010): TermoStat. Version 3.0. Accédé en ligne le 13 novembre 2017, <http:// termostat.ling.umontreal.ca/>.

6. «Un seuil d'acceptabilité permet d'exclure des mots ou expressions non considérés comme terminologiques dans le texte». Voir le guide de l'utilisateur de TermoStat. Consulté le 13 novembre 2017, <http://termostat.ling.umontreal.ca/doc_termostat/doc_termostat.html>.

7. Pour le tableau complet des données quantitatives extraites du corpus, consultez l'Annexe 2 à la fin de cet article.

8. Nous trouvons de la variation dénominative dans le nombre de composantes pour expliquer la même information. Pour ce genre de cas, plusieurs points de vue existent en ce qui concerne les conséquences cognitives de la variation dénominative en question. En effet, comme FernándezSilva l'a souligné après la lecture d'une version antérieure du présent article faite en 2017, il n’y aurait pas de conséquences cognitives dans ce type de variation, contrairement à ce qui est proposé par Freixa (2002). Nous ne considérons pas non plus qu'il y ait des conséquences cognitives à cette variation formelle dans le cas du couple museo ambiental et museo de ambiente.

9. Comme pour le cas précédent de museo ambiental et museo de ambiente, nous ne considérons pas que la variation formelle entre le couple d'unités museo online et museo en línea ait des conséquences cognitives.

\section{RÉFÉRENCES}

CABré, Maria Teresa (1999): La terminología. Representación y comunicación. Elementos para una teoría de base comunicativa y otros artículos. Barcelona: IULA/UPF. 
CABré, Maria Teresa (2000): Sur la représentation mentale des concepts: bases pour une tentative de modélisation. In: Henri BÉjoInt et Philippe Thorron, dir. Le sens en terminologie. Lyon: Presses universitaires de Lyon, 20-39.

CABRÉ, Maria Teresa (2002): Teorías de la terminología: de la prescripción a la descripción. In: Giovanni Adamo et Valeria Della Valle, Actes de Innovazione lessicale e terminologie specialistiche nella società del plurilinguismo. Florence: Leo S. Olschki, 169-188.

Cabré, Maria Teresa (2008): El principio de poliedricidad: la articulación de lo discursivo, lo cognitivo y lo lingüístico en Terminología (I). IBÉRICA. 16:9-36.

Ciapuscio, Guiomar (1998): La terminología desde el punto de vista textual: selección, tratamiento y variación. Organon. 12(26):15 p. Consulté le 31 mai 2018, <https://seer.ufrgs.br/ organon/article/view/29558>.

Condamines, Anne et Rebeyrolle, Josette (1996): Point de vue en langue spécialisée. Meta. 42(1):174-184.

Diki-Kidiri, Marcel, dir. (2008): Le vocabulaire scientifique dans les langues africaines - Pour une approche culturelle de la terminologie. Paris: Karthala.

Drouin, Patrick (2003): Term extraction using non-technical corpora as a point of leverage. Terminology. 9(1):99-117.

FAber, Pamela et León-Araúz, Pilar (2014): Specialized knowledge dynamics: From cognition to culture-bound terminology. In: Rita Temmerman et Marc Van Campenhoudt, dir. Dynamics and Terminology. An interdisciplinary perspective on monolingual and multilingual culture-bound communication. Amsterdam/Philadelphie: John Benjamins, 135-158.

Faber, Pamela, Montero Martínez, Silvia, Castro Prieto, María Rosa, et al. (2006): Processoriented terminology management in the domain of Coastal Engineering. Terminology. 12(2):189-213.

Fernández-Silva, Sabela (2013) : Punto de vista y variación denominativa. Debate Terminológico. 9:11-37.

Fernández-Silva, Sabela (2016): The cognitive and rhetorical role of term variation and its contribution to knowledge construction in research articles. Terminology. 22(1):52-79.

FreIXA, Judit (2002): La variació terminològica: anàlisi de la variació denominativa en textos de diferent grau d'especialització de l'àrea de medi ambient. Barcelona: IULA-UPF.

Freixa, Judit (2005): Variación terminológica: ¿por qué y para qué? Meta. 50(4):CD-ROM.

FreiXa, Judit (2006): Causes of denominative variation in terminology: A typology proposal. Terminology. 12(1):51-77.

GaUdin, François (2003): Socioterminologie. Une approche sociolinguistique de la terminologie. Champs linguistiques. Bruxelles: Duculot.

GAUDIN, François (2005): La socioterminologie. Langages. 2005/1(157):81-93.

Joan Casademont, Anna et Lorente Casafont, Mercè (2005): Variation sémantique dans un vocabulaire lié au monde des musées: multilinguisme et déséquilibre. In: Groupe de travail Terminologie et Intelligence Artificielle, ed. Actes de la conférence TIA-2005. (6 ${ }^{\text {èmes }}$ rencontres «Terminologie et intelligence artificielle», Université de Rouen, Rouen, 4-5 avril 2005). Rouen: Laboratoire DYALANG, 105-114.

Joan Casademont, Anna et Lorente Casafont, Mercè (2006a) : Variación semántica y conocimiento especializado: un caso para el vocabulario de la museística. In: Maria JuAnGarau, Marian Amengual Pizarro et Joana Salazar Noguera, dir. Lingüística aplicada en la sociedad de la información y la comunicación. (XXIII congreso de la Asociación Española de Lingüística Aplicada, Universitat de les Illes Balears, Palma de Mallorca, 10-12 mars 2005). Palma de Mallorca: Servei de Publicacions i Intercanvi Científic de la Universitat de les Illes Balears, 133-142.

Joan Casademont, Anna et Lorente Casafont, Mercè (2006b) : Variación semántica y conocimiento especializado: un caso para el vocabulario de la museología. RdM. Revista de Museología: Publicación científica al servicio de la comunidad museológica. 35:30-35.

Kostina, Irina (2011): Clasificación de la variación conceptual de los términos basada en la modulación semántica discursiva. Íkala, revista de lenguaje y cultura. 16(27):35-73. 
Kostina, Irina (2014): Variación conceptual de los términos en el discurso especializado. Barcelona: IULA-UPF.

Pecman, Mojca (2012): Tentativeness in Term Formation. A Study of Neology as a Rethorical Device in Scientific Papers. Terminology. 18(1):62-80.

Pecman, Mojca (2014): Variation as a Cognitive Device. How Scientists Construct Knowledge throught Term Formation. Terminology. 20(1):1-24.

PÉRALDI, Sandrine (2012): L'anglais de spécialité en chimie organique: entre indétermination terminologique et multidimensionnalité. ASp. 62:5-24.

SAN MARTín, Antonio (2016): La representación de la variación contextual mediante definiciones terminológicas flexibles. Thèse de doctorat non publiée. Grenade: Universidad de Granada.

Seghezzi, Natalia (2013): Variación terminológica: de la escritura a la oralidad. Debate Terminológico. 9:62-80.

TeBÉ, Carles (2005): La representació conceptual en terminologia. L'atribució temàtica en els bancs de dades terminològiques. Barcelona: IULA-UPF.

Temmerman, Rita (2000): Towards new ways of terminology description: The sociocognitiveapproach. Amsterdam/Philadelphie: John Benjamins.

TERCEDOR, María Isabel (2004): Descripción y variación de la representación terminológica: el caso de la dimensión tipos de cáncer. In: Pamela Faber et Catalina Jiménez Hurtado, dir. Investigar en terminología. Grenade: Comares, 199-214.

Tercedor Sánchez, Maribel (2013): Una perspectiva situada de la variación denominativa. Debate Terminológico. 9:81-88.

\section{AnNexes}

\section{Annexe 1: Corpus espagnol (documents):}

\begin{tabular}{|c|c|c|}
\hline $\begin{array}{l}\text { Références bibliographiques - } \\
18 \text { textes - 2006-2015 }\end{array}$ & Origine & $\begin{array}{l}\text { Mots } \\
\text { (après } \\
\text { nettoyage) }\end{array}$ \\
\hline $\begin{array}{l}\text { Azor Lacasta, Ana (2012): El Programa Ibermuseos y los Encuentros } \\
\text { iberoamericanos de museos como herramientas de cooperación. Museos.es: } \\
\text { Revista de la Subdirección General de Museos Estatales. 7-8:292-297. }\end{array}$ & $\begin{array}{l}\text { Espagne } \\
\text { (Madrid) }\end{array}$ & 2837 \\
\hline $\begin{array}{l}\text { Baena Alcántara, María Dolores (2012): El Museo Arqueológico de } \\
\text { Córdoba. Un museo en transformación. Museos.es: Revista de la Subdirección } \\
\text { General de Museos Estatales. 7-8:308-319. }\end{array}$ & $\begin{array}{l}\text { Espagne } \\
\text { (Madrid) }\end{array}$ & 4163 \\
\hline $\begin{array}{l}\text { CARrobles SAntos, Jesús (2012): Los museos de Toledo. Reflexiones para un } \\
\text { proyecto. Museos.es: Revista de la Subdirección General de Museos Estatales. } \\
\text { 7-8:198-215. }\end{array}$ & $\begin{array}{l}\text { Espagne } \\
\text { (Madrid) }\end{array}$ & 7300 \\
\hline $\begin{array}{l}\text { DeL CAMPo CAÑzares, Elpidio (2012): Diseño expositivo y museografía } \\
\text { didáctica en los espacios de arte. ASRI: Arte y Sociedad, Revista de } \\
\text { Investigación. } 1 \text {. }\end{array}$ & $\begin{array}{l}\text { Espagne } \\
\text { (Séville/ } \\
\text { Malaga) }\end{array}$ & 5748 \\
\hline $\begin{array}{l}\text { Fernández SABUgo, Mar (2012): El museo durmiente. Museos.es: Revista de } \\
\text { la Subdirección General de Museos Estatales. 7-8:192. }\end{array}$ & $\begin{array}{l}\text { Espagne } \\
\text { (Madrid) }\end{array}$ & 484 \\
\hline $\begin{array}{l}\text { GÁNDARA VÁzQUEZ, Manuel (2013): Reseña de libro - Interacción e } \\
\text { interactividad: Manual de museografía Interactiva. Intervención. 4(7):75-77. }\end{array}$ & $\begin{array}{l}\text { Mexique } \\
\text { (Mexico) }\end{array}$ & 1720 \\
\hline $\begin{array}{l}\text { IzQUIERdo Peraile, Isabel et García Arnillas, Salvador (2012): El Museo } \\
\text { Arqueológico de Asturias (Oviedo): identidad y memoria del patrimonio } \\
\text { asturiano. Museos.es: Revista de la Subdirección General de Museos Estatales. } \\
\text { 7-8:264-279. }\end{array}$ & $\begin{array}{l}\text { Espagne } \\
\text { (Madrid) }\end{array}$ & 6072 \\
\hline $\begin{array}{l}\text { LADRERo CABALlERo, Tomás (2015): Enmarcación y museografía en el } \\
\text { Museo del Prado en las últimas décadas del siglo XIX. Libros de la corte. } \\
\text { 10:7-29. }\end{array}$ & $\begin{array}{l}\text { Espagne } \\
\text { (Madrid) }\end{array}$ & 7034 \\
\hline $\begin{array}{l}\text { López, Olga (2012): La reforma integral del Museu Marítim de Barcelona: } \\
\text { una renovación de la historia marítima a través de la museografía. Revista de } \\
\text { Estudios Marítimos del País Vasco. 7:631-643. }\end{array}$ & $\begin{array}{l}\text { Espagne } \\
\text { (San } \\
\text { Sebastián) }\end{array}$ & 8372 \\
\hline $\begin{array}{l}\text { López Benito, María Victoria (2013): La Museografía de los Museos de } \\
\text { Arte: un Modelo en Proceso de Cambio. Anales de Historia del Arte. } \\
\text { 13:461-470. }\end{array}$ & $\begin{array}{l}\text { Espagne } \\
\text { (Madrid) }\end{array}$ & 4173 \\
\hline
\end{tabular}


López Benito, María Victoria (2014): Estudio exploratorio sobre la interpretación didáctica del arte en el museo a través de tecnologías móviles. Thèse de doctorat non publiée. Barcelone: Universitat de Barcelona, 27-70. Nuevo Gómez, Alejandro et Moreno Conde, Margarita (2012): Con voz de mujer: Patrimonio en Femenino, primera exposición en línea de la Red Digital de Colecciones de Museos de España. Museos.es: Revista de la Subdirección General de Museos Estatales. 7-8:298-307.

Padilla Blanco, Blanca (2012): Museo del Greco. El proceso de renovación de una institución centenaria. Museos.es: Revista de la Subdirección General de Museos Estatales. 7-8:280-291.

Plaza, Carlos (2014): Palladio Museum: historia y museografía para un nuevo museo-taller sobre la arquitectura de Andrea Palladio. Revista PH. 85:15-17.

RodríGUez SÁNCHEz, Keilyn (2011): Estudios de visitantes a museos. Actualidades Investigativas en Educación. 11(2):1-37.

SAGAste, Delia (2006): La gestión de las colecciones de arte asiático en los museos españoles: el caso de la Colección Palacio en el Museo de Bellas Artes de Bilbao. In: Pedro SAn Ginés Aguilar, dir. La investigación sobre Asia Pacífico en España. Grenade: Universidad de Granada, 455-472.

Sibina, Joan (2015): La museografía de la felicidad. Una museografía accesible para todos. Museos.es: Revista de la Subdirección General de Museos Estatales. 9-10:98-109

Tejera Pinilla, Carmen (2013): Investigación didáctica: la cibermuseografía didáctica como contexto educativo para la enseñanza y el aprendizaje del patrimonio. Estudio de páginas web educativas de museos virtuales de arte. Clío. 39:30 p. Consulté le 4 avril 2018, <http://clio.rediris.es/ n39/articulos/Tejera.pdf $>$.

$$
\text { TOTAL }
$$

\begin{tabular}{|c|c|}
\hline $\begin{array}{c}\text { Espagne } \\
\text { (Barcelone) }\end{array}$ & 17025 \\
$\begin{array}{c}\text { Espagne } \\
\text { (Madrid) }\end{array}$ & 5235 \\
\hline $\begin{array}{c}\text { Espagne } \\
\text { (Madrid) }\end{array}$ & 5001 \\
\hline $\begin{array}{c}\text { Espagne } \\
\text { (Séville) }\end{array}$ & 1306 \\
\hline $\begin{array}{c}\text { Costa Rica } \\
\text { (San José) }\end{array}$ & 9468 \\
\hline $\begin{array}{c}\text { Espagne } \\
\text { (Granada) }\end{array}$ & 7266 \\
\hline $\begin{array}{c}\text { Espagne } \\
\text { (Madrid) }\end{array}$ & 3324 \\
\hline $\begin{array}{c}\text { Espagne } \\
\text { (Séville) }\end{array}$ & 6522 \\
\hline
\end{tabular}

\section{ANNEXE 2}

Données quantitatives de l'extraction de TermoStat pour muse(o)-:

\begin{tabular}{|c|c|c|c|}
\hline Candidat & \multicolumn{2}{|c|}{ Fr. corpus } & Exemples \\
\hline \multicolumn{4}{|r|}{ museo } \\
\hline museo & \multicolumn{2}{|c|}{1158} & \\
\hline$\underline{\text { museo }}+$ adjectif & 51 & \multirow[t]{4}{*}{143} & $\begin{array}{l}\text { museo virtual, museo nacional, museo arqueológico, } \\
\text { museo digital... }\end{array}$ \\
\hline$\underline{\text { museo }}+$ préposition + nom & 45 & & 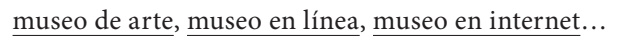 \\
\hline$\underline{\text { museo }}+$ nom & 19 & & 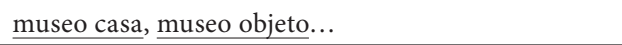 \\
\hline nom + préposition $+\underline{\text { museo }}$ & 27 & & $\begin{array}{l}\text { montaje de museo arqueológico, inauguración del } \\
\text { museo sefardí, red de museos públicos... }\end{array}$ \\
\hline \multicolumn{4}{|c|}{ museístico } \\
\hline museístico & \multicolumn{2}{|l|}{1} & \\
\hline nom $+\underline{\text { museístico }}$ & 42 & \multirow[t]{2}{*}{48} & $\begin{array}{l}\text { práctica museística, espacio museístico, institución } \\
\text { museística... }\end{array}$ \\
\hline $\begin{array}{l}\text { nom }+ \text { préposition }+ \text { nom }+ \\
\text { museístico }\end{array}$ & 6 & & $\begin{array}{l}\text { conjunto de equipamientos museísticos, tipo de } \\
\underline{\text { experiencia museística... }}\end{array}$ \\
\hline \multicolumn{4}{|c|}{ museal et dérivés } \\
\hline nom $+\underline{\text { museal }}$ & \multicolumn{2}{|l|}{14} & espacio museal, medio museal... \\
\hline nom + musealizado & \multicolumn{2}{|l|}{3} & yacimientos musealizados \\
\hline musealización & \multicolumn{2}{|l|}{3} & \\
\hline$\frac{\text { musealización }}{+ \text { nom }}+$ préposition & \multicolumn{2}{|l|}{1} & $\underline{\text { musealización del territorio }}$ \\
\hline musealizar & \multicolumn{2}{|l|}{1} & \\
\hline
\end{tabular}




\begin{tabular}{|c|c|c|c|}
\hline \multicolumn{4}{|c|}{ museología } \\
\hline$\underline{\text { museología }}$ & \multicolumn{2}{|l|}{21} & \\
\hline préfixe + museología & \multicolumn{2}{|l|}{2} & cibermuseología \\
\hline museología + adjectif & \multicolumn{2}{|l|}{1} & museología contemporánea \\
\hline $\begin{array}{l}\text { nom }+ \text { préposition }+ \\
\text { museología }\end{array}$ & \multicolumn{2}{|l|}{2} & revista de museología, curso de museología \\
\hline nom + museología & \multicolumn{2}{|l|}{1} & web museología \\
\hline \multicolumn{4}{|c|}{ museólogo } \\
\hline museólogo & \multicolumn{2}{|l|}{8} & \\
\hline$\frac{\text { museólogo }}{\text { nom }}+$ préposition + & \multicolumn{2}{|l|}{1} & $\underline{\text { museólogos de corte }}$ \\
\hline $\begin{array}{l}\text { nom }+ \text { préposition }+ \\
\text { museólogo }\end{array}$ & \multicolumn{2}{|l|}{2} & esfera de museólogos... \\
\hline \multicolumn{4}{|c|}{ museológico } \\
\hline nom $+\underline{\text { museológico }}$ & \multicolumn{2}{|l|}{46} & $\begin{array}{l}\text { programa museológico, materias museológicas, } \\
\text { discurso museológico... }\end{array}$ \\
\hline $\begin{array}{l}\text { nom }+ \text { préposition }+ \text { nom }+ \\
\text { museológico }\end{array}$ & \multicolumn{2}{|l|}{8} & $\begin{array}{l}\text { preservación del patrimonio museológico, } \\
\text { articulación de políticas museológicas... }\end{array}$ \\
\hline \multicolumn{4}{|c|}{ museografía } \\
\hline$\underline{\text { museografía }}$ & \multicolumn{2}{|l|}{94} & \\
\hline préfixe $+\underline{\text { museografía }}$ & \multicolumn{2}{|l|}{21} & cibermuseografía, cibermuseografía didáctica \\
\hline museografía + adjectif & 40 & \multirow{2}{*}{48} & 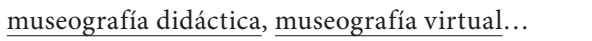 \\
\hline$\frac{\text { museografía }}{\text { nom }}+$ préposition + & 8 & & $\underline{\text { museografía de arte... }}$ \\
\hline $\begin{array}{l}\text { nom }+ \text { préposition }+ \\
\text { museografía }\end{array}$ & \multicolumn{2}{|l|}{5} & $\frac{\text { modelo de museografía interactiva, }}{\text { museografía didáctica.... }}$ \\
\hline \multicolumn{4}{|c|}{ museógrafo } \\
\hline museógrafo & \multicolumn{2}{|l|}{6} & \\
\hline \multicolumn{4}{|c|}{ museográfico } \\
\hline museográfico & \multicolumn{2}{|l|}{9} & \\
\hline nom $+\underline{\text { museográfico }}$ & 105 & \multirow[t]{2}{*}{124} & $\begin{array}{l}\text { diseño museográfico, didáctica museográfica, } \\
\text { discurso museográfico... }\end{array}$ \\
\hline $\begin{array}{l}\text { nom }+ \text { préposition }+ \text { nom }+ \\
\text { museográfico }\end{array}$ & 19 & & $\begin{array}{l}\text { tipo de propuestas museográficas, fase de proyecto } \\
\text { museográfico... }\end{array}$ \\
\hline
\end{tabular}

